\title{
Stability of metabolically healthy obesity over 8 years: the English Longitudinal Study of Ageing
}

\author{
Mark Hamer ${ }^{1,2}$, Joshua A Bell'2, Severine Sabia², G David Batty² and Mika Kivimäki² \\ ${ }^{1}$ National Centre for Sport and Exercise Medicine, School of Sport, Exercise and Health Sciences, Loughborough \\ University, Loughborough, LE11 3TU, UK and ${ }^{2}$ Department of Epidemiology and Public Health, University College \\ London, London, UK
}

Correspondence should be addressed to M Hamer

Email

m.hamer@lboro.ac.uk

\begin{abstract}
Objective: Metabolically healthy obesity possibly reflects a transitional stage before the onset of metabolic dysfunction, but few studies have characterised this transition. We examined the behavioural and biological characteristics of healthy obese adults that progressed to an unhealthy state over 8 years follow-up.

Methods: Participants were 2422 men and women (aged 63.3 \pm 7.7 years, $44.2 \%$ men) from the English Longitudinal Study of Ageing. Obesity was defined as BMI $\geq 30 \mathrm{~kg} / \mathrm{m}^{2}$. Based on blood pressure (BP), HDL-cholesterol, triglycerides, HbA1c and C-reactive protein (CRP) participants were classified as 'healthy' ( 0 or 1 metabolic abnormality) or 'unhealthy' ( $\geq 2$ metabolic abnormalities).

Results: Over 8 years follow-up, $44.5 \%$ of healthy obese subjects had transitioned into an unhealthy state, compared to only 16.6 and $26.2 \%$ of healthy normal-weight and overweight adults respectively. Compared with healthy obese adults who remained stable, those who progressed to an unhealthy state were more likely to have high $\mathrm{BP}(75.0 \%$ vs $37.0 \%$, age- and sex-adjusted odds ratio (OR) 8.9, 95\% Cl 4.7-17.0), high CRP (53.7\% vs $17.0 \%$, OR=8.6, 95\% Cl 4.1-18.0), high $\mathrm{HbA} 1 \mathrm{c}(46.3 \%$ vs $5.9 \%, \mathrm{OR}=13.8,95 \% \mathrm{Cl} 6.1-31.2)$ and high triglycerides $(45.4 \%$ vs $11.9 \%, \mathrm{OR}=5.9,95 \% \mathrm{Cl} 2.9-12.0)$ at follow-up, with excess risk remaining independent of lifestyle factors including self-reported physical activity. Progression to an unhealthy state was also linked with significant gains in waist circumference $(B=2.7,95 \% \mathrm{Cl}, 0.5-4.9 \mathrm{~cm})$.
\end{abstract}

Conclusion: These data show that a healthy obesity phenotype is relatively unstable. Transition to an unhealthy state is characterised by multiple biological changes that are not fully explained by lifestyle risk factors.

\section{Introduction}

Population-based studies have identified an obese phenotype that is not accompanied by adiposity-associated cardio-metabolic risk factors. Termed 'metabolically healthy obesity,' it is unclear if the healthy obese phenotype is a stable trait or a transitional stage prior to the onset of metabolic dysfunction (1). This instability may explain the inconsistency in findings between studies with shorter and longer follow-up periods in relation to healthy obesity and the risk of incident type 2 diabetes and cardiovascular disease $(2,3)$.
In particular, the characteristics of healthy obese participants that remain stable or develop metabolic abnormalities are poorly understood. In a recent cohort study, $67 \%$ of healthy obese adults maintained stable metabolic health profiles at 4 years follow-up, and these adults displayed lower waist circumference at baseline although no differences in physical activity, alcohol or smoking were observed (4). In the Whitehall II study of British men and women, more than half of healthy obese adults progressed to an unhealthy obese state after

Published by Bioscientifica Ltd. 
20 years (5). The aim of the present study was to examine stability of healthy obesity over an 8-year follow-up period and to describe the metabolic and lifestyle profile of obese participants that progressed to an unhealthy state.

\section{Subjects and methods}

\section{Study sample and procedures}

The English Longitudinal Study of Ageing (ELSA) is an ongoing cohort study that contains a nationally representative sample of free-living men and women born on or before 29 February 1952 (6). Data collected at wave 2 (2004-2005) were used as the baseline for present analyses, as this was the first occasion clinical information was gathered. A clinical assessment was repeated 8 years later. Participants gave full informed written consent to participate in the study and ethical approval was obtained from the London Multi-Centre Research Ethics Committee.

\section{Clinical measurements at baseline and follow-up}

Nurses collected anthropometric data (weight, height, waist circumference), blood pressure (BP) and non-fasting blood samples using standard protocols at baseline and follow-up. Body weight was measured using Tanita electronic scales without shoes and in light clothing, and height was measured using a Stadiometer with the Frankfort plane in the horizontal position (Seca Leicester stadiometer, Birmingham, UK). BMI was calculated as weight $(\mathrm{kg}) /$ height $(\mathrm{m})$ squared. Waist circumference was recorded twice mid-way between the iliac crest and lower rib using measuring tape. An average of the first two measurements was used provided these differed by no more than $3 \mathrm{~cm}$; otherwise, a third reading was taken and the two closest results utilised. Systolic and diastolic BP was measured with an Omron HEM-907 BP monitor three times in the sitting position after 5 -min rest between each reading. The initial reading was discarded and an average of the second and third BP recordings was used for the present analyses. Blood samples were analysed for C-reactive protein (CRP), HDL cholesterol, triglycerides and HbA1c. Detailed information on the technicalities of the blood analysis, the internal quality control and the external quality assessment for the laboratory have been described elsewhere (7). Additional data were collected on physician-diagnosed conditions (hypertension, diabetes) and medication use.

\section{Lifestyle risk factors at baseline}

Health-related questions included cigarette smoking (current, previous or non-smoker), the frequency of participation in light, moderate and vigorous physical activities (more than once per week, once per week, one to three times per month, hardly ever), and the frequency of alcohol intake (daily, 5-6/weeks, 3-4/weeks, $1-2 /$ weeks, $1-2 /$ month, once every couple of months, $1-2 /$ years, never).

\section{Statistical analyses}

We used the conventional criteria to define obesity (BMI $\geq 30 \mathrm{~kg} / \mathrm{m}^{2}$ ) and being overweight (BMI $\geq 25$ and $\left.<30 \mathrm{~kg} / \mathrm{m}^{2}\right)$. A healthy metabolic status was based on existing criteria (8) and, according to availability of data, defined as having less than two of the following metabolic risk factors: high $\mathrm{BP}$ ( $\mathrm{BP} \geq 130 / 85 \mathrm{mmHg}$, or hypertension diagnosis, or use of anti-hypertensive medication), impaired glycaemic control (HbA1c $>6.0 \%(42.1 \mathrm{mmol} / \mathrm{mol})$ or doctor's diagnosed diabetes), systemic inflammation (CRP $\geq 3 \mathrm{mg} / \mathrm{l})$, low HDL cholesterol $(<1.03 \mathrm{mmol} / \mathrm{l}$ in men and $<1.30 \mathrm{mmol} / \mathrm{l}$ in women) and high triacylglycerol $(\geq 1.7 \mathrm{mmol} / \mathrm{l})$. Participants were then categorized into four groups: 'healthy non-obese,' 'unhealthy non-obese,' 'healthy obese,' and unhealthy obese.' We categorised healthy obese participants as those that met criteria for healthy obesity both at baseline and at 8 years follow-up ('stable healthy obese') and those that developed $\geq 2$ metabolic risk factors (unstable healthy obese). Differences in baseline characteristics between stable and unstable groups were tested using ANOVA and $\chi^{2}$ tests. Metabolic profiles at follow-up between stable and unstable groups were compared using logistic regression and general linear models to examine individual risk factors as categorical and continuous variables respectively. In multivariable models we adjusted effect estimates for several covariates in a step-wise fashion: model 1 contained age, sex and baseline risk factor; model 2 contained additional behavioural and anthropometric covariates, including baseline smoking, alcohol, physical activity and change in central obesity (waist circumference). We also examined the concept of 'weight cycling,' which was defined as participants who experienced both weight gain ( $>5 \%$ gain in $\mathrm{BMI})$ and weight loss $(>5 \%$ reduction BMI) between examinations (9). For these analyses we utilised data collected from baseline (wave 2; 2004-2005), wave 4 (2008-2009) and wave 6 (2012-2013). Analyses were conducted using SPSS version 20. 


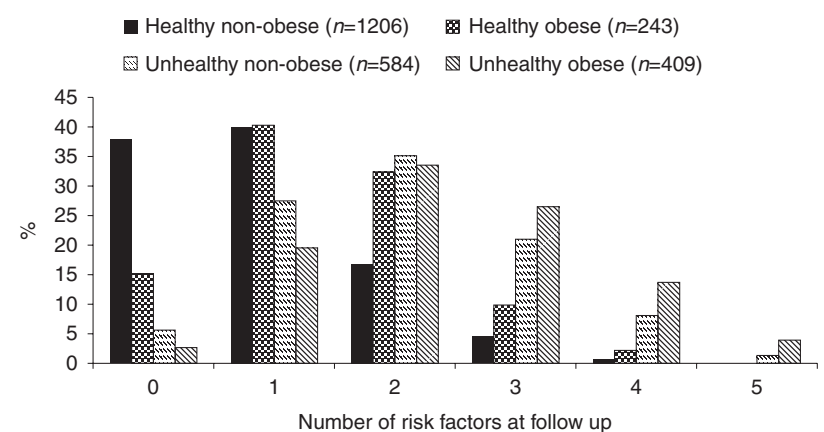

Figure 1

Progression of metabolic risk factors over 8 years follow-up.

\section{Results}

At baseline the sample consisted of 3851 individuals, although loss to follow-up resulted in a final analytic sample of 2442 men and women (aged 63.3 \pm 7.7 years, $44.2 \%$ men). Participants excluded (drop-outs) did not differ in age, gender, or BMI, but were less sphysically active $(23.8$ vs $13.5 \%, P<0.001)$ and more likely to be metabolically unhealthy ( 46.2 vs $40.6 \% \geq 2$ risk factors,
$P=0.001)$ compared with included participants respectively.

At baseline, 1206 participants were classified as healthy non-obese, 584 as unhealthy non-obese, 243 as healthy obese, and 389 as unhealthy obese. At follow-up, $44.5 \%$ of baseline healthy obese adults had transitioned into an unhealthy state, compared with $22 \%$ of healthy non-obese adults (Fig. 1). We further categorised the nonobese into 'healthy normal weight' $(n=530)$ and 'healthy overweight' ( $n=676) ; 16.6$ and $26.2 \%$ respectively had transitioned into an unhealthy state at follow up. Adjusting for age and sex, healthy obese adults were four times as likely to transition into an unhealthy state compared with healthy normal weight adults (odds ratio $(\mathrm{OR})=4.00$, 95\% CI, 2.81-5.69). As expected, this association was weaker when the healthy obese were compared with the healthy overweight (OR $=2.30,95 \%$ CI, 1.69-3.13). This likelihood remained unchanged after further adjustment for baseline lifestyle risk factors, including self-reported physical activity, in relation to healthy normal weight $(\mathrm{OR}=3.79,95 \% \mathrm{CI}, 2.60-5.51)$ or overweight $(\mathrm{OR}=2.30,95 \% \mathrm{CI}, 1.66-3.19)$ as the reference category. At baseline, 656 participants $(n=70$ healthy

Table 1 Characteristics of healthy obese adults at baseline who remained healthy (stable) or became unhealthy (unstable) at follow-up.

Baseline characteristics

Age (years, \pm s.D.)

Men (\%)

Vigorous physical activity at least once/week (\%)

Current smoker (\%)

At least one alcoholic drink/week (\%)

Body mass index $\left(\mathrm{kg} / \mathrm{m}^{2}\right)$

Waist circumference $(\mathrm{cm})$

Systolic BP $(\mathrm{mmHg})$

Diastolic BP $(\mathrm{mmHg})$

HDL-cholesterol ( $\mathrm{mmol} / \mathrm{l})$

Triacylglycerol $(\mathrm{mmol} / \mathrm{l})$

C-reactive protein (log unit)

HbA1C (\%)

Zero metabolic risk factors (\%)

Weight cycling

Metabolic risk factors at follow-up ${ }^{a}$

High blood pressure (\%)

Impaired glycaemic control (\%)

Low HDL-C (\%)

High triglycerides (\%)

Inflammation (\%)

\begin{tabular}{c}
$\begin{array}{c}\text { Stable healthy obese } \\
(\boldsymbol{n}=\mathbf{1 3 5})\end{array}$ \\
\hline $61.9 \pm 7.1$ \\
40.0 \\
30.4 \\
7.4 \\
70.0 \\
$32.7 \pm 2.8$ \\
$102.9 \pm 13.2$ \\
$132.2 \pm 14.2$ \\
$77.1 \pm 8.7$ \\
$1.59 \pm 0.31$ \\
$1.41 \pm 0.65$ \\
$1.09 \pm 0.57$ \\
$5.35 \pm 0.32$ \\
35.6 \\
12.7
\end{tabular}

\begin{tabular}{c}
$\begin{array}{c}\text { Unstable healthy obese } \\
(\boldsymbol{n}=\mathbf{1 0 8})\end{array}$ \\
\hline $62.4 \pm 7.4$ \\
44.4 \\
37.0 \\
13.0 \\
65.3 \\
$32.8 \pm 3.3$ \\
$104.9 \pm 13.5$ \\
$134.9 \pm 14.9$ \\
$76.4 \pm 7.7$ \\
$1.48 \pm 0.28$ \\
$1.65 \pm 0.68$ \\
$1.19 \pm 0.59$ \\
$5.51 \pm 0.28$ \\
20.4 \\
13.2
\end{tabular}

37.0
5.9
0.7
11.9
17.0

75.0

46.3

11.1

45.4

53.7

\begin{tabular}{c}
\hline P value \\
\hline 0.59 \\
0.49 \\
0.54 \\
0.15 \\
0.62 \\
0.73 \\
0.24 \\
0.16 \\
0.49 \\
0.005 \\
0.005 \\
0.19 \\
0.001 \\
0.01 \\
0.92 \\
Odds ratio (95\% Cl) \\
(stable (ref) vs unstable) \\
$8.9(4.7,17.0)$ \\
$13.8(6.1,31.2)$ \\
$15.9(1.9,132.5)$ \\
$5.9(2.9,12.0)$ \\
$8.6(4.1,18.1)$ \\
\end{tabular}

${ }^{a}$ High blood pressure (clinic BP $\geq 130 / 85 \mathrm{mmHg}$, or hypertension diagnosis, or use of anti-hypertensive medication), impaired glycaemic control (HbA1c $>6.0 \%$ or doctor-diagnosed diabetes), systemic inflammation (C-reactive protein $\geq 3 \mathrm{mg} / \mathrm{l}$ ), low $\mathrm{HDL}$ cholesterol $(<1.03 \mathrm{mmol} / \mathrm{l}$ in $\mathrm{men}$ and $<1.30 \mathrm{mmol} / \mathrm{l}$ in women) and high triacylglycerol $(\geq 1.7 \mathrm{mmol} / \mathrm{l})$.

bodds ratio (OR) for having each risk factor for stable (ref) vs unstable healthy obese adults; adjusted for age, sex and baseline risk factor. 
obese) had exceptional metabolic health (zero risk factors; $10.7 \%$ of obese adults vs $32.7 \%$ of non-obese adults). At follow-up, 325 (55.5\%) of healthy non-obese and 19 (27.1\%) of healthy obese remained free of any risk factors.

There were no differences in demographic and behavioural characteristics between stable and unstable healthy obese adults at baseline, although a higher proportion of stable participants had zero risk factors and stable healthy obese displayed higher baseline levels of HDL-cholesterol, lower triacylglycerol and HbA1c (Table 1). At follow-up, all metabolic risk factors were more prevalent among unstable healthy obese compared with stable participants. Compared with healthy obese adults that remained stable, those that progressed to an unhealthy state also gained greater waist circumference at follow up $(B=2.7 ; 95 \% \mathrm{CI}, 0.5,4.9 \mathrm{~cm})$, although no increase in BMI was observed $(B=0.63 ;-0.02$, $1.28 \mathrm{~kg} / \mathrm{m}^{2}$ ) after baseline adjustment. In the overall cohort, $9.3 \%$ of study members were identified as weight cycling,' although this was not associated with stability of metabolic health. Lifestyle risk factors remained similar at follow-up between stable and unstable healthy obese; for example, participation in vigorous physical activity (25.8\% vs $31.1 \%, P=0.43)$ and smoking (2.3\% vs $5.7 \%$, $P=0.33)$ was comparable in stable and unstable participants respectively at follow-up. When we compared the characteristics of stable and unstable healthy overweight participants, the results were largely similar to those presented for the healthy obese group.

Unstable healthy obese adults demonstrated higher HbA1c $(B=0.22 ; 0.14,0.30 \%)$, higher triglycerides $(B=0.31 ; 0.16,0.47 \mathrm{mmol} / \mathrm{l})$, higher CRP $(B=0.34 ; 0.20$, $0.47 \log$ units) and lower HDL cholesterol $(B=-0.12$; $-0.19,-0.06 \mathrm{mmol} / \mathrm{l})$ at follow-up after baseline adjustments for each respective risk factor. When we adjusted these models for change in waist circumference, the associations with $\mathrm{HbA1c}$ were marginally attenuated $(B=0.19 ; 0.12$, 0.27) but effect estimates for other biomarkers were unchanged. Further adjustments for lifestyle risk factors at baseline or follow-up did not influence the associations (data available on request).

We further examined the characteristics of unhealthy obese adults at baseline who remained unhealthy or became healthy at follow-up (Table 2). At follow-up, $23.1 \%$ of unhealthy obese had transitioned into a healthy state. There were few differences in baseline characteristics between those that remained unhealthy and became healthy, except that participants transitioning into a healthy state were more likely to consume alcohol regularly (Table 2). Compared with unhealthy obese adults that became healthy, those that remained unhealthy gained greater waist circumference $(B=5.5 ; 95 \% \mathrm{CI}, 3.3$, $7.7 \mathrm{~cm})$ and BMI $\left(B=1.3 ; 0.5,2.0 \mathrm{~kg} / \mathrm{m}^{2}\right)$ at follow-up after baseline adjustment.

Table 2 Characteristics of unhealthy obese adults at baseline who remained unhealthy or became healthy at follow-up.

\begin{tabular}{|c|c|c|c|}
\hline Baseline characteristics & $\begin{array}{l}\text { Remained unhealthy at } \\
\text { follow-up }(n=299)\end{array}$ & $\begin{array}{l}\text { Became healthy at follow-up } \\
\qquad(n=90)\end{array}$ & $P$ value \\
\hline Age (years, \pm s.D.) & $62.3 \pm 7.1$ & $63.0 \pm 7.1$ & 0.38 \\
\hline Men $(\%)$ & 39.5 & 34.4 & 0.39 \\
\hline $\begin{array}{l}\text { Vigorous physical activity at least } \\
\text { once/week (\%) }\end{array}$ & 22.7 & 26.7 & 0.73 \\
\hline Current smoker (\%) & 7.2 & 10.3 & 0.59 \\
\hline At least one alcoholic drink/week (\%) & 45.8 & 60.5 & 0.03 \\
\hline Body mass index $\left(\mathrm{kg} / \mathrm{m}^{2}\right)$ & $34.3 \pm 3.7$ & $33.5 \pm 3.7$ & 0.10 \\
\hline Waist circumference $(\mathrm{cm})$ & $107.5 \pm 16.0$ & $104.9 \pm 8.3$ & 0.15 \\
\hline Weight cycling (\%) & 9.8 & 7.5 & 0.54 \\
\hline Metabolic risk factors at follow-up ${ }^{a}$ & & & $\begin{array}{c}\text { Odds ratio }(95 \% \mathrm{Cl})^{\mathrm{b}} \\
\text { (Healthy (ref) vs } \\
\text { unhealthy) }\end{array}$ \\
\hline High blood pressure (\%) & 85.3 & 52.2 & $6.3(3.3,12.2)$ \\
\hline Impaired glycaemic control (\%) & 62.5 & 7.8 & $13.5(5.5,13.0)$ \\
\hline Low HDL-C (\%) & 24.4 & 2.2 & $16.8(3.9,72.7)$ \\
\hline High triglycerides (\%) & 56.5 & 7.8 & $20.9(9.2,47.8)$ \\
\hline Inflammation (\%) & 54.5 & 17.8 & $7.5(4.0,13.8)$ \\
\hline
\end{tabular}

${ }^{a}$ High blood pressure (clinic BP $\geq 130 / 85 \mathrm{mmHg}$, or hypertension diagnosis, or use of anti-hypertensive medication), impaired glycaemic control (HbA1c $>6.0 \%$ or doctor-diagnosed diabetes), systemic inflammation (C-reactive protein $\geq 3 \mathrm{mg} / \mathrm{l}$ ), low $\mathrm{HDL}$ cholesterol $(<1.03 \mathrm{mmol} / \mathrm{l}$ in $\mathrm{men}$ and $<1.30 \mathrm{mmol} / \mathrm{l}$ in women) and high triglycerides ( $\geq 1.7 \mathrm{mmol} / \mathrm{l})$.

${ }^{b}$ Odds ratio (OR) for having each risk factor for remaining healthy (ref) vs becoming unhealthy obese adults, adjusted for age, sex and baseline risk factor. 


\section{Discussion}

The present study has several key findings. First, healthy obesity is a relatively unstable phenotype. Second, instability in healthy obesity was not attributable to selfreported lifestyle behaviours including physical activity, but we did observe greater increases in waist circumference that is likely to be reflective of adverse changes in visceral adiposity. Third, important features of instability were also development of low grade systemic inflammation, impaired glycaemic control, and reduction in HDL-cholesterol.

Our findings relating to stability in healthy obesity over time are largely consistent with the limited available evidence $(4,5)$. However, when one considers defining metabolic health as 'zero risk factors,' we observed striking results; first, obese adults without any metabolic risk factors are rare (10.7\% of obese participants); and second, obese adults who maintain zero risk factors over time are rarer still (2.9\% of obese participants).

Only one previous study has examined the characteristics of stable and unstable healthy obese adults. Consistent with our findings, they showed no differences in self-reported physical activity, alcohol or smoking (4), although the unstable healthy obese demonstrated higher waist circumference at baseline. Our results add to this evidence base by showing that unstable healthy obese adults developed greater increases in central adiposity and several metabolic risk factors over 8 years follow-up, one particularly notable factor being impaired glycaemic control. The unstable healthy obese displayed slightly elevated HbA1c levels at baseline compared with their stable counterparts, but also greater increases in this risk factor at follow up as our analyses were adjusted for baseline. Previous work has also demonstrated that weight cycling is associated with metabolic syndrome $(9,10)$, although this finding was not replicated in our data, possibly owing to limited follow-up of body mass assessments over the 8 years. Several overfeeding studies have demonstrated that healthy obese adults display different biological responses to moderate weight gain, such as increased adipose tissue capacity for lipogenesis (11) and favourable characteristics of subcutaneous adipose tissue that help prevent visceral fat depots (12). Thus, more subtle differences in metabolic function that were not possible to measure in this study may differentiate stable and unstable healthy obesity.

Several limitations should be noted. Participants with poorer metabolic health at baseline were less likely to complete follow-up assessments. Thus, the proportion of unhealthy participants that transitioned into a healthy metabolic state at follow-up may be over-estimated. The use of self-report measures to assess lifestyle risk factors may have introduced bias. Indeed, we recently demonstrated differences in physical activity between healthy and unhealthy obese adults when using objective data but not for self-reporting (13). Thus, the results on self-reported physical activity in the present study may underestimate the associations, although a major bias is unlikely.

In summary, healthy obesity is a relatively unstable phenotype at high risk of developing vascular, inflammatory, glycaemic and lipidemic abnormalities over time. A true healthy obese phenotype capable of maintaining exceptional metabolic health (zero risk factors) for a prolonged period appears to be exceptionally rare in the general population. Disease prevention strategies are therefore required in the healthy obese.

\section{Declaration of interest}

The authors declare that there is no conflict of interest that could be perceived as prejudicing the impartiality of the research reported.

\section{Funding}

The data was made available through the UK Data Archive. ELSA was developed by a team of researchers based at University College London, the Institute of Fiscal Studies and the National Centre for Social Research. The funding was provided by the National Institute on Ageing in the United States (grants 2R01AG7644-01A1 and 2R01AG017644) and a consortium of UK government departments coordinated by the Office of National Statistics. J A Bell is supported by an Economic and Social Research Council (ESRC) studentship. S Sabia is supported by the NIH NIA (R01AG034454) and ESRC (ES/J023299/1). M Kivimäki is supported by the Medical Research Council (MR/K013351/1), the National Heart, Lung and Blood Institute (R01HL36310), the National Institute of Aging (NIA) (R01AG034454), NordForsk (75021) and an ESRC professorial fellowship (ES/J023299/1). The funders had no role in the study design; in the collection, analysis and interpretation of data; in writing of the report; or in the decision to submit the paper for publication. The developers and funders of ELSA do not bear any responsibility for the analyses or interpretations presented here.

\section{Author contribution statement}

M Hamer had full access to the data and takes responsibility for the integrity and accuracy of the results. M Hamer drafted the paper. All authors contributed to the concept and design of the study and critical revision of the manuscript.

\section{References}

1 Stefan N, Häring HU, Hu FB \& Schulze MB. Metabolically healthy obesity: epidemiology, mechanisms, and clinical implications. Lancet. Diabetes \& Endocrinology 20131 152-162. (doi:10.1016/S22138587(13)70062-7) 
2 Kramer CK, Zinman B \& Retnakaran R. Are metabolically healthy overweight and obesity benign conditions? A systematic review and meta-analysis Annals of Internal Medicine 2013159 758-769. (doi:10.7326/0003-4819-159-11-201312030-00008)

3 Fan J, Song Y, Chen Y, Hui R \& Zhang W. Combined effect of obesity and cardio-metabolic abnormality on the risk of cardiovascular disease: a meta-analysis of prospective cohort studies. International Journal of Cardiology 2013168 4761-4768. (doi:10.1016/j.ijcard.2013.07.230)

4 Appleton SL, Seaborn CJ, Visvanathan R, Hill CL, Gill TK, Taylor AW, Adams RJ \& North West Adelaide Health Study Team . Diabetes and cardiovascular disease outcomes in the metabolically healthy obese phenotype: a cohort study. Diabetes Care 201336 2388-2394. (doi:10.2337/dc12-1971)

5 Bell JA, Hamer M, Sabia S, Singh-Manoux A, Batty GD \& Kivimaki M. The natural course of healthy obesity over 20 years. Journal of the American College of Cardiology 201565 101-102. (doi:10.1016/j.jacc. 2014.09.077)

6 Steptoe A, Breeze E, Banks J \& Nazroo J. Cohort profile: the English longitudinal study of ageing. International Journal of Epidemiology 2013 42 1640-1648. (doi:10.1093/ije/dys168)

7 Graig R, Deverill C \& Pickering K. Quality control of blood, saliva and urine analytes. In Health Survey for England 2004, Methodology and Documentation, vol 2, pp 34-41. Eds K Spronston \& J Mindell. London: The Information Centre, 2006.

8 Wildman RP, Muntner P, Reynolds K, McGinn AP, Rajpathak S, WylieRosett J \& Sowers MR. The obese without cardiometabolic risk factor clustering and the normal weight with cardiometabolic risk factor clustering: prevalence and correlates of 2 phenotypes among the US population (NHANES 1999-2004). Archives of Internal Medicine 2008 168 1617-1624. (doi:10.1001/archinte.168.15.1617)

9 Fung MD, Canning KL, Mirdamadi P, Ardern CI \& Kuk JL. Lifestyle and weight predictors of a healthy overweight profile over a 20-year follow-up. Obesity 201523 1320-1325. (doi:10.1002/oby.21087)

10 Petersmarck K, Teitelbaum HS, Bond JT, Bianchi L, Hoerr SM \& Sowers MF. The effect of weight cycling on blood lipids and blood pressure in the Multiple Risk Factor Intervention Trial Special Intervention Group. International Journal of Obesity and Related Metabolic Disorders 199923 1246-1255. (doi:10.1038/sj.ijo.0801058)

11 Fabbrini E, Yoshino J, Yoshino M, Magkos F, Tiemann Luecking C, Samovski D, Fraterrigo G, Okunade AL, Patterson BW \& Klein S. Metabolically normal obese people are protected from adverse effects following weight gain. Journal of Clinical Investigation 2015125 787-795. (doi:10.1172/JCI78425)

12 Alligier M, Gabert L, Meugnier E, Lambert-Porcheron S, Chanseaume E, Pilleul F, Debard C, Sauvinet V, Morio B, Vidal-Puig A et al. Visceral fat accumulation during lipid overfeeding is related to subcutaneous adipose tissue characteristics in healthy men. Journal of Clinical Endocrinology and Metabolism 201398 802-810. (doi:10.1210/ jc.2012-3289)

13 Bell JA, Hamer M, van Hees VT, Singh-Manoux A, Kivimaki M \& Sabia S. Healthy obesity and objective physical activity. American Journal of Clinical Nutrition 2015102 268-275. (doi:10.3945/ajcn.115.110924)

Received 1 May 2015

Revised version received 1 August 2015

Accepted 18 August 2015 